Polymer Journal, Vol. 38, No. 11, pp. 1165-1172 (2006)

(C) 2006 The Society of Polymer Science, Japan

\title{
Effect of Interfacial Reaction and Morphology on Rheological Properties of Reactive Bilayer
}

\author{
Hwang Yong KIM, Hye Jeong KIM, and Jin Kon KIm ${ }^{\dagger}$ \\ National Creative Research Center for Block Copolymer Self-Assembly, Department of Chemical Engineering \\ and Polymer Research Institute, Pohang University of Science \& Technology, Kyungbuk 790-784, Korea
}

(Received July 31, 2006; Accepted August 25, 2006; Published October 11, 2006)

\begin{abstract}
We investigated the effect of interfacial reaction and morphology on the rheological properties of a reactive bilayer polymer system consisting of end-functionalized polystyrene with carboxylic acid (PS-mCOOH) and poly(methyl methacrylate-ran-glycidylmethacrylate) (PMMA-GMA). We found that the complex viscosity $\left(\left|\eta^{*}\right|\right)$ of the reactive bilayer was unusually higher than the predicted value from the inverse relationship which holds for nonreactive bilayer, even though the interfacial roughness generated from PMMA-graft-PS copolymers formed in situ from the reaction near the interface was much smaller than the thicknesses of two plates of PS-mCOOH and PMMA-GMA. This indicates that in addition to the effect of the improved mechanical strength or interfacial mixing between chains by the presence of graft copolymers located near the interface, the roughness of the interface greatly influences $\left|\eta^{*}\right|$ of the reactive bilayer. [doi:10.1295/polymj.PJ2006086]

KEY WORDS Rheological Properties / Reactive Polymer Bilayer / Interfacial Roughness /
\end{abstract}

Polymer blends consisting of two or more polymers have been developed to exhibit desirable physical and mechanical properties. ${ }^{1,2}$ Because all polymer blends must be processed to be used for the final product, the rheological properties of polymer blends are very important. For the long times, the rheological properties of polymer blends have been extensively studied experimentally. ${ }^{3-14}$ Also, many kinds of mixing rules for the prediction of the viscosity of polymer blends from the viscosity of the constituent components have been introduced in the literature. ${ }^{15-17}$ Among the many predictions, additive (eq 1a and eq $1 \mathrm{~b}$ ) or inverse additive relationships (eq $2 \mathrm{a}$ and eq $2 \mathrm{~b}$ ) have been widely used for the prediction of the viscosity of a polymer blend $\left(\eta_{b}\right) .{ }^{15-17}$

$$
\begin{aligned}
& \eta_{b}=\phi_{1} \eta_{1}+\phi_{2} \eta_{2} \\
& \log \eta_{b}=\phi_{1} \log \eta_{1}+\phi_{2} \log \eta_{2} \\
& \frac{1}{\eta_{b}}=\frac{\phi_{1}}{\eta_{1}}+\frac{\phi_{2}}{\eta_{2}} \\
& \frac{1}{\log \eta_{b}}=\frac{\phi_{1}}{\log \eta_{1}}+\frac{\phi_{2}}{\log \eta_{2}}
\end{aligned}
$$

where $\phi_{i}$ and $\eta_{i}$ are the volume faction and the viscosity of component $i$, respectively.

On the other hand, polymer multilayers have been employed for a polymer waveguide or biomaterials. ${ }^{18-20}$ Thus, the rheological properties of the multilayered polymer film would be important to assess their processability. Among many predictions described above, the viscosity of a bilayer polymer system without interfacial slip is commonly given by the inverse relationship given by eq $2 \mathrm{a}^{21,22}$ Zhao and Macosko ${ }^{23}$ showed that the viscosity of a multilayer polymer system $\left(\eta_{\text {total }}\right)$ consisting of two immiscible polymers could be expressed as:

$$
\frac{1}{\eta_{\text {total }}}=\frac{\sum_{i=1}^{N} h_{1, i}}{H \eta_{1}}+\frac{\sum_{j=1}^{N} h_{2, j}}{H \eta_{2}}
$$

where $h_{k, i}(k=1,2)$ is the $i$-th layer thickness of component $k$, and $H$ is total thickness of multilayer. This equation becomes eq $2 \mathrm{a}$ because of $\sum_{i=1}^{N} h_{k, i} / H=\phi_{\mathrm{k}}$.

Recently, we ${ }^{24}$ reported that the complex viscosity $\left(\left|\eta^{*}\right|\right)$ for a reactive bilayer consisting of end-functionalized polystyrene with carboxylic acid (PS-mCOOH) and poly(methyl methacrylate-ran-glycidyl methacrylate) (PMMA-GMA) is unusually larger than the predicted value by eq $2 \mathrm{a}$. A large increase in $\left|\eta^{*}\right|$ was also found for other reactive bilayers consisting of polyamide 6 (PA6)/polyethylene grafted with glycidyl methacrylate ${ }^{20}$ and PA6/poly(styrene-co-maleic anhydride). ${ }^{25}$ These results indicate that $\left|\eta^{*}\right|$ of a reactive polymer bilayer could not be decided by that of pure polymer component (or mixing rule), suggesting that another important parameters must be considered. Since a reactive polymer bilayer generates graft or block copolymers near the interface, each block (or graft) component can entangle (or stitch) easily with the corresponding homopolymer located near the interface. Thus, the interface should be strengthened compared with the interface without graft or block

${ }^{\dagger}$ To whom correspondence should be addressed (Tel: +82-54-279-2276, Fax: +82-54-279-8298, E-mail: jkkim@postech.ac.kr). 
copolymers. This could increase the viscosity of the reactive polymer bilayer compared with a non-reactive polymer bilayer. Also, as the roughness of the interface of a bilayer increases, the additional friction force against the flow increases because the roughened interface would be normal to the flow direction; thus the viscosity increases. It is noted that the friction force arising from the roughened interface normal to the flow direction is larger than that parallel to the flow direction.

In this study, we investigated in detail the effect of the interfacial reaction and roughness of the interface on $\left|\eta^{*}\right|$ of reactive polymer bilayer consisting of PS-mCOOH and PMMA-GMA. It is noted that with increasing the reaction time the amount of in situ formed PMMA-graft-PS copolymer increases, and in turn the interface becomes roughened. We found that even if the interfacial roughness in the reactive bilayer is quite small (less than $0.1 \%$ of the thickness of two plates of PS-mCOOH and PMMA-GMA), this roughness significantly increases the viscosity of the reactive bilayer.

\section{EXPERIMENTAL}

\section{Materials and Sample Preparation}

An end-functionalized polystyrene with carboxylic acid (PS-mCOOH) was purchased from Aldrich Chem. Co. A poly(methyl methacrylate-ran-glycidylmethacrylate) (PMMA-GMA) was synthesized via free radical polymerization. A PS homopolymer with similar viscosity to that of PS-mCOOH was also purchased from Aldrich Chem. Co. The molecular characteristics of the polymers employed in this study are given in Table I.

The reaction between the carboxylic acid in PS$\mathrm{mCOOH}$ and the epoxy group in PMMA-GMA occurs easily at elevated temperatures, ${ }^{26-33}$ giving rise to in situ formation of PMMA-graft-PS copolymers. We prepared the plates of PS-mCOOH, PMMA-GMA and PS homopolymer by using compression molding on Si-wafer to obtain smooth surfaces of the plates, and then annealed at $130{ }^{\circ} \mathrm{C}$ for $24 \mathrm{~h}$. The PS-mCOOH (or PS) plate was placed on the top of the PMMAGMA plate. The thickness of each plate was varied from 0.3 to $1 \mathrm{~mm}$.

Table I. Molecular characteristics of polymers employed in this study

\begin{tabular}{lcccc}
\hline \multicolumn{1}{c}{ Samples } & $\begin{array}{c}M_{\mathrm{w}} \\
(\mathrm{g} / \mathrm{mol})\end{array}$ & $M_{\mathrm{w}} / M_{\mathrm{n}}$ & $\begin{array}{c}\eta_{\mathrm{o}} \text { at } 180^{\circ} \mathrm{C} \\
(\mathrm{Pa} \mathrm{s})\end{array}$ & Functionality \\
\hline PS-mCOOH & 135,000 & 1.13 & $8 \times 10^{3}$ & 1 \\
PMMA-GMA & 115,500 & 1.7 & $2.5 \times 10^{5}$ & 12.5 \\
PS & 155,000 & 1.4 & $8 \times 10^{3}$ & 0 \\
\hline
\end{tabular}

\section{Rheological Measurements}

After two plates of PS-mCOOH and PMMA-GMA were placed inside the $25 \mathrm{~mm}$ parallel-plate fixture of a rheometer (Advanced Rheometric Expansion System, TA Instruments) at $180^{\circ} \mathrm{C}$ under a nitrogen atmosphere, variations of dynamic storage modulus $\left(G^{\prime}\right)$ and dynamic loss modulus $\left(G^{\prime \prime}\right)$ were monitored with time at strain amplitude $\left(\gamma_{0}\right)$ of 0.005 and angular frequency $(\omega)$ of $0.1 \mathrm{rad} / \mathrm{s}$. As we reported in our previous study, ${ }^{24}$ the interfacial morphology and reaction were essentially independent of $\gamma_{0}$ for small values of $\gamma_{0}(<0.005)$. The absolute value of complex viscosity $\left(\left|\eta^{*}\right|\right)$ was obtained from the following relationship: $\left|\eta^{*}\right|=\left[\left(G^{\prime} / \omega\right)^{2}+\left(G^{\prime \prime} / \omega\right)^{2}\right]^{1 / 2}$. The temperature control was satisfactory to within $\pm 1^{\circ} \mathrm{C}$. All rheological measurements were conducted by using a 200 FRTN1 transducer with a lower limit of $0.08 \mathrm{~g} \mathrm{~cm}$.

\section{The Roughness of Interfacial Morphology}

To investigate interfacial morphology and measure the degree of roughening, the specimen was removed from the parallel-plate fixture and then quenched in ice water. Subsequently, the PS plate was completely removed by selectively dissolving solvent of cyclohexane at $40^{\circ} \mathrm{C}$ for $35 \mathrm{~h}$. The morphology of the interface was obtained by atomic force microscopy (AFM) (Digital Instrument; D3000) with silicon nitride tips on cantilevers (Nanoprobe) in the tapping mode. The root-mean square (rms) roughness of the interface was calculated as given in our previous study. ${ }^{33}$

\section{Prevention of the Interchain Mixing Between Graft Copolymers and Homopolymers}

To obtain the effect of the interfacial roughness alone on the $\left|\eta^{*}\right|$ of the reactive bilayer, the interchain mixing between the polymer chains of graft copolymers and the corresponding homopolymers was prevented by introducing an osmium tetraoxide $\left(\mathrm{OsO}_{4}\right)$ layer between the reactive bilayer. For this purpose, the reaction between PMMA-GMA/PS-mCOOH bilayer was first carried out and unreacted PS-mCOOH was removed by selective rinsing with acetic acid. Then, $\mathrm{OsO}_{4}$ coating was performed for $30 \mathrm{~s}$ using a coater (Meiwa Shoji Co.; NEOC-ST). The thickness of $\mathrm{OsO}_{4}$ layer was below $5 \mathrm{~nm}$; thus, this layer does not affect on the viscosity and roughness of the final bilayer. Subsequently, PS plate was placed on the $\mathrm{OsO}_{4}$-coated PMMA-GMA plate on which PMMAgraft-PS copolymers were formed, and the viscosity measurement was carried out.

\section{The Effect of Pre-made Graft Copolymers Layer on the Viscosity}

To distinguish the effect of pre-made graft copolymer and in situ formed graft copolymer on the $\left|\eta^{*}\right|$ of 
the bilayer, a PMMA-graft-PS copolymer layer was placed between the two plates. For this purpose, PMMA-graft-PS copolymers are produced by the reaction between PMMA-GMA and PS- $\mathrm{mCOOH}$, as given in ref 33. To facilitate the floating the graft copolymer from silicon wafer, silicon oxide with $\sim 100 \mathrm{~nm}$ thickness was evaporated onto a silicon wafer. $^{34,35}$ The PMMA-g-PS copolymer was spincoated from toluene solutions onto the wafer and then annealed at $170{ }^{\circ} \mathrm{C}$ under vacuum for two days, and quenched to room temperature. The thickness of the graft copolymer layer varied from 50 to $200 \mathrm{~nm}$. The films were floated onto the surface of a $5 \mathrm{wt} \%$ HF solution, transferred to a water bath, then transferred to PMMA plate.

\section{RESULTS AND DISCUSSION}

Figure 1 gives the variations of $\left|\eta^{*}\right|$ of the symmetric $(\mathrm{PS}-\mathrm{mCOOH}) /(\mathrm{PMMA}-\mathrm{GMA})$ bilayer with reaction time at $\gamma_{0}=0.005$ and $\omega=0.1 \mathrm{rad} / \mathrm{s}^{24}$

The thickness of the PS-mCOOH $\left(\mathrm{H}_{P S-m C O O H}\right)$ and PMMA-GMA $\left(\mathrm{H}_{P M M A-G M A}\right)$ plates was the same $(0.3 \mathrm{~mm})$; thus, the total bilayer thickness $\left(\mathrm{H}_{\text {total }}\right)$ was $0.6 \mathrm{~mm}$. Three different regimes are clearly distinguished in Figure 1. At stage I, the sharp interface begins to undulate, because PMMA-graft-PS copolymers are formed from the chemical reaction between PS-mCOOH and PMMA-GMA at the interface. At stage II, $\left|\eta^{*}\right|$ does not increase with reaction time, because the reactive polymer chain ends could not diffuse through the densely-packed graft copolymer layer formed at stage I. At the final stage III, the reactive polymer chains penetrate again into the densely-

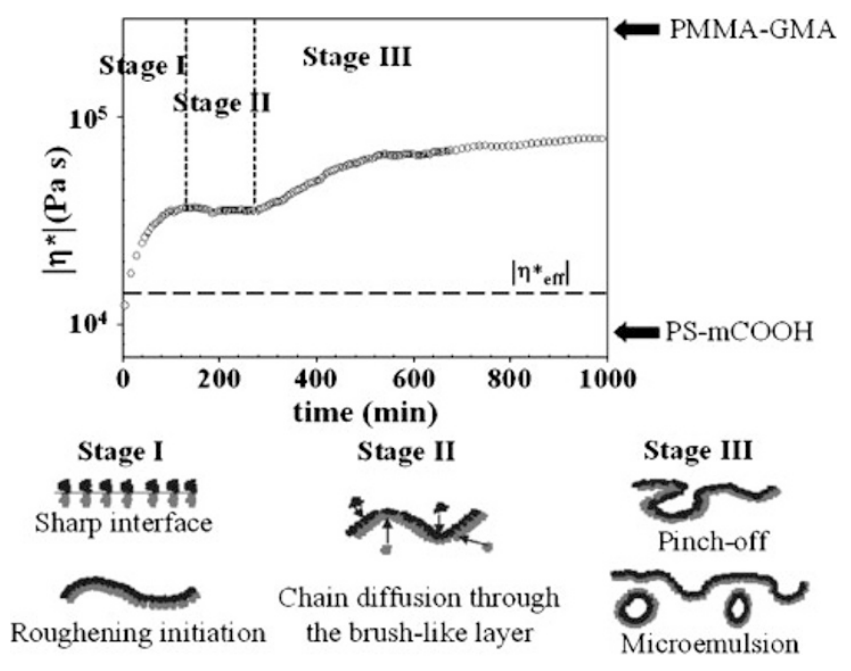

Figure 1. Plot of $\left|\eta^{*}\right|$ at $\omega=0.1 \mathrm{rad} / \mathrm{s}$ and $180^{\circ} \mathrm{C}$ versus reaction time for symmetric PS-mCOOH/PMMA-GMA bilayer and schematic describing variations of interfacial morphology. The thickness of each plate is $0.3 \mathrm{~mm}$. The dashed line is the predicted $\left|\eta^{*}\right|$ by eq 2 a. packed graft copolymer layers and then undergo further chemical reactions. The interfacial morphologies corresponding to each of the three stages are given schematically in the lower part of Figure $1 .{ }^{24}$

In Figure 1, the predicted $\left|\eta^{*}\right|$ by eq $2 \mathrm{a}$ is given as a dashed line, from which the $\left|\eta^{*}\right|$ of the reactive bilayer at a reaction time of $16 \mathrm{~h}$ was unusually larger than the predicted value. In this study, we consider following effects to explain the large increase of $\left|\eta^{*}\right|$ for the reactive bilayer: 1) entanglement (or stitching) between graft copolymer and homopolymer chains, 2) additional friction force generated by the roughened interface, and 3) the interfacial relaxation.

The contribution of the interfacial relaxation to the viscosity increase is roughly estimated by the emulsion model: ${ }^{36,37}$

$$
\left|\eta^{*}\right|=\left|\eta_{m}^{*}\right| \frac{1+3 \phi H}{1-2 \phi H}
$$

in which $\left|\eta^{*}\right|$ and $\left|\eta_{m}{ }^{*}\right|$ are the complex viscosity of the emulsion and medium phase (PS homopolymer), respectively, and $\phi$ is the volume fraction of the dispersed phase (PMMA homopolymer). $H$ is a function of the ratio of viscosity (or shear moduli) of dispersed phase to medium phase, the interfacial tension, and the dispersed domain size. For PMMA/PS blend where the $\left|\eta^{*}\right|$ of PMMA is much larger than that of PS (see Figure 1), $H$ in eq 4 becomes 0.5 . For a bilayer, $\phi$ is approximated expressed by the interfacial roughness (a maximum value of $\sim 200 \mathrm{~nm}$ ) divided by the total specimen thickness $(0.6 \mathrm{~mm})$, because the emulsions would be considered near the interface. Thus, the expected increase in $\left|\eta^{*}\right|$ by the interfacial relaxation is at most $\sim 0.08 \%$, which is negligible in the large increase in $\left|\eta^{*}\right|$, as seen in Figure 1.

Since PMMA-graft-PS copolymer was formed in situ near the interface, PMMA (or PS) chains in the graft copolymer can entangle with the corresponding PMMA (or PS) homopolymer located near the interface. Although we could not determine the exact thickness of the entanglement near the interface, this region contributes differently to the viscosity of a reactive bilayer compared with neat PMMA-GMA or PS-mCOOH plates located far from the interface. Hereafter, this region is referred to as the third layer. It should be noted that the third layer has nothing related to a region where PMMA-GMA and PS$\mathrm{mCOOH}$ polymer chains can inter-diffuse into the interface, but related to a region where rheological properties (or mechanical strength) are different from those of neat polymers. Namely, the third layer consists of in situ formed PMMA-graft-PS copolymers and parts of PMMA-GMA and PS-mCOOH layers which are affected by the presence of graft copolymers. 
Since the third layer is assumed to be treated as another independent layer, one can attempt to estimate the $\left|\eta^{*}\right|$ of the reactive layer by extension of eq $2 \mathrm{a}$ :

$$
\begin{aligned}
\frac{1}{\eta_{\text {total }}(t)}= & \frac{\phi_{P S-m \mathrm{COOH}}(t)}{\eta_{P S-m \mathrm{COOH}}}+\frac{\phi_{P M M A-G M A}(t)}{\eta_{P M M A-G M A}} \\
& +\frac{\phi_{\text {third-layer }}(t)}{\eta_{\text {third-layer }}}
\end{aligned}
$$

in which $\phi_{P S-m C O O H}(t)$ (or $\left.\phi_{P M M A-G M A}(t)\right)$ and $\phi_{\text {third-layer }}(t)$ are the volume fractions of PS-mCOOH (or PMMA-GMA) and the third layer at a given reaction time $\mathrm{t}$. It is noted that $\phi_{\text {third-layer }}(t)$ should depend on reaction time, because the increase of the amount of in situ formed PMMA-graft-PS copolymer (and thus the increase of the thickness of third layer) enhances the entanglement (or stitching) effect. Notice that $\phi_{P S-m C O O H}(t)$ is not the same as $\mathrm{H}_{P S-m C O O H} /$ $\mathrm{H}_{\text {total }}$, but might be expressed by $\mathrm{H}_{P S-m C O O H} / \mathrm{H}_{\text {total }}-$ $\phi_{\text {third-layer }} / 2$. Similarly, $\phi_{P M M A-G M A}(t)$ in eq 5 is given by $\mathrm{H}_{P M M A-G M A} / \mathrm{H}_{\text {total }}-\phi_{\text {third-layer }}(t) / 2$, and $\phi_{\text {third-layer }}(t)=\mathrm{H}_{\text {third-layer }} / \mathrm{H}_{\text {total }}$. This is because PS (or PMMA) chains in the graft copolymers are assumed to locate at the half of the thickness of the third layer, and entangle with PS-mCOOH (or PMMAGMA) homopolymer chains.

It is seen in Figure 1 that the value of $\left|\eta^{*}\right|$ before the reaction is $\sim 1.5 \times 10^{4} \mathrm{Pas}$, which is very close to the prediction by eq 5 with $\phi_{\text {third-layer }}(0)=0$. This indicates that an inverse relationship of $\left|\eta^{*}\right|$ (eq 2a) could be valid for the prediction of a reactive polymer bilayer before the reaction (or without any in situ graft copolymer). On the other hand, the $\left|\eta^{*}\right|$ of the reactive bilayer at a reaction time of $16 \mathrm{~h}$ was $\sim 8 \times 10^{4} \mathrm{Pas}$. To predict this large value of $\left|\eta^{*}\right|$ by eq $5, \phi_{P S-m C O O H}$ $(1,000 \mathrm{~min})$ should be $\sim 0.17$ (thus, $\phi_{\text {third-layer }}(1,000$ min) $\sim 0.66$ ), because $\eta_{\text {third-layer }}$ and $\eta_{P M M A-G M A}$ are much larger than $\eta_{P S-m \mathrm{COOH}}$. Namely, the thickness of the third layer $\left(\mathrm{H}_{\text {third-layer }}\right)$ should be $\sim 0.4 \mathrm{~mm}$, which is about 2,000 times larger than the rms roughness $(\sim 180 \mathrm{~nm})$ of the interface for this reactive bilayer at a reaction time of $16 \mathrm{~h}$. This result led us to consider that the large increase in the $\left|\eta^{*}\right|$ is not due to the unusually large thickness of the third layer (or the entanglement effect of PMMA-graft-PS copolymers with PMMA-GMA (or PS-mCOOH) homopolymer near the interface).

Therefore, we consider that the second effect (additional friction force generated by the roughened interface) greatly affect the $\left|\eta^{*}\right|$ of the reactive bilayer. It is noted that the roughness of the interface of this bilayer becomes very large $(\sim 180 \mathrm{~nm})$ at a long reaction time. ${ }^{24}$ This roughened interface can act as an obstacle to the flow since the flow direction is normal to the roughened interface. To verify in detail which of two effects (the entanglement effect and the additional
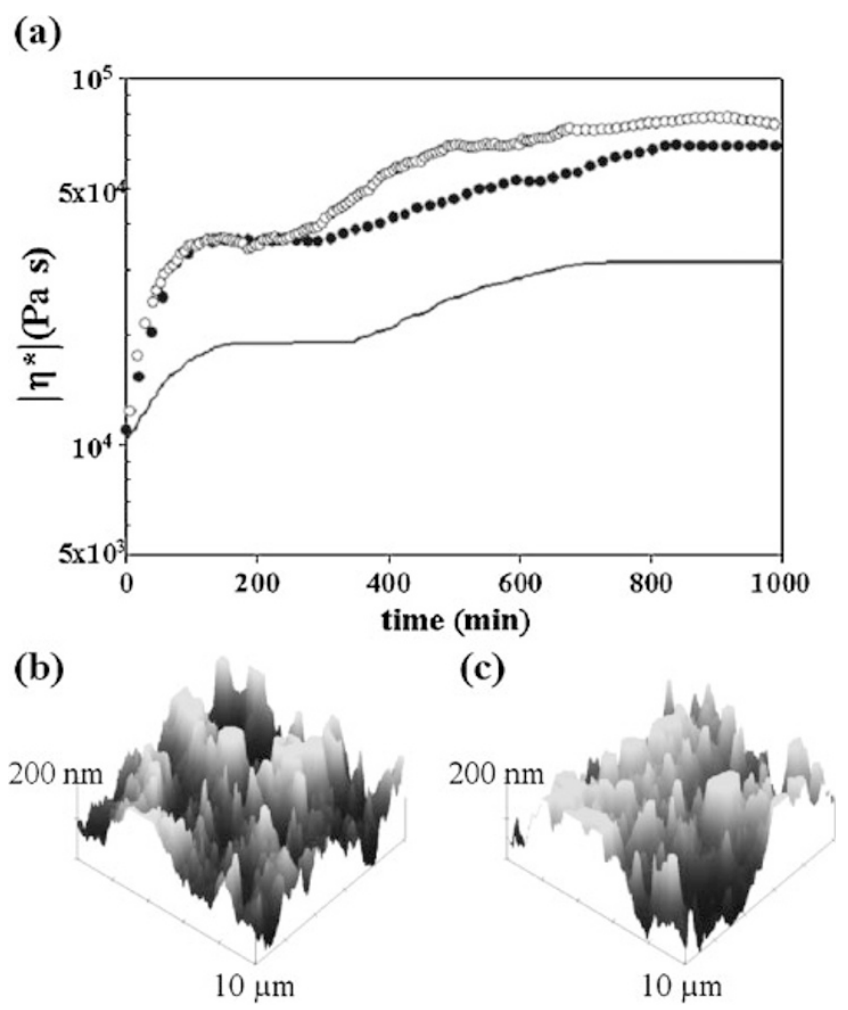

Figure 2. (a) Plot of $\left|\eta^{*}\right|$ at $\omega=0.1 \mathrm{rad} / \mathrm{s}$ and $180^{\circ} \mathrm{C}$ versus reaction time for symmetric PS-mCOOH/PMMA-GMA bilayer with total thickness of $2.0 \mathrm{~mm}(\bullet)$. The solid line is the estimated $\left|\eta^{*}\right|$ by eq 5 for the bilayer with total thickness of $2.0 \mathrm{~mm}$. For a reference, a plot of $\left|\eta^{*}\right|$ versus reaction time for symmetric PS$\mathrm{mCOOH} / \mathrm{PMMA}-\mathrm{GMA}$ bilayer with total thickness of $0.6 \mathrm{~mm}$ is added (O). (b) and (c) are the AFM images for the symmetric bilayers with total thickness of $2.0 \mathrm{~mm}$ and $0.6 \mathrm{~mm}$, respectively, after reacted for $16 \mathrm{~h}$ at $180^{\circ} \mathrm{C}$. The rms roughness of these two thicknesses was essentially the same $(\sim 180 \mathrm{~nm})$.

friction force) becomes more important to decide the $\left|\eta^{*}\right|$ of the reactive bilayer, we carried out an experiment by changing the thickness of the total bilayer $\left(\mathrm{H}_{\text {total }}\right)$ from 0.6 to $2.0 \mathrm{~mm}$. Figure $2 \mathrm{a}$ gives the change of $\left|\eta^{*}\right|$ with reaction time for two reactive bilayers with the same thickness of two plates $\left(\mathrm{H}_{\text {total }}=2 \mathrm{~mm}\right.$ and $\left.0.6 \mathrm{~mm}\right)$.

It is seen that before the reaction, $\left|\eta^{*}\right|$ for both bilayers are essentially same. This is attributed to the fact that although measured torque (or frictional force) is smaller for the thicker bilayer than that for the thinner one because of the large amount of the soft material (PS plate) in the former, $\left|\eta^{*}\right|$ expressed by toque multiplied by total bilayer thickness would not change much. We also found that the increase of $\left|\eta^{*}\right|$ with reaction time for these two bilayers was almost the same. This suggests that eq 5 could not be used for the determination of $\left|\eta^{*}\right|$ for a reactive bilayer. If eq 5 had been valid, $\left|\eta^{*}\right|$ at a given reaction time for the thicker bilayer should have been smaller than that for the thinner bilayer, as schematically shown in 


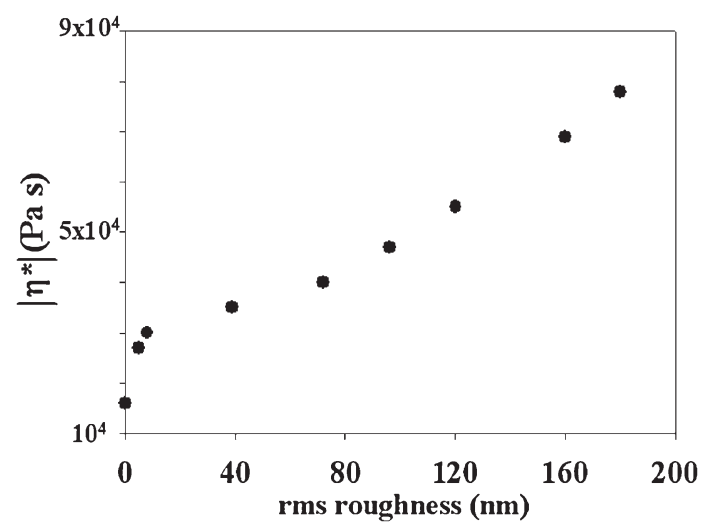

Figure 3. Plot of $\left|\eta^{*}\right|$ at $\omega=0.1 \mathrm{rad} / \mathrm{s}$ and $180^{\circ} \mathrm{C}$ versus rms roughness for symmetric PS-mCOOH/PMMA-GMA bilayer with total thickness of $0.6 \mathrm{~mm}$.

solid line in Figure 2. Namely, $\phi_{\text {third-layer }}$ at $1000 \mathrm{~min}$ for the thicker layer should be smaller that that for the latter. This is because $\mathrm{H}_{\text {total }}$ for the thicker bilayer is 3.3 times larger than that for the thinner layer, whereas the $\mathrm{H}_{\text {third-layer }}$ for the two bilayers would be almost the same, since the rms roughness of two bilayers at the reaction time of $16 \mathrm{~h}$ is the same $(\sim 180 \mathrm{~nm})$, as demonstrated in Figure 2b. A small difference in $\left|\eta^{*}\right|$ between two symmetric bilayers at longer reaction times might be due to different total thickness where the stitching effect behaves differently. Therefore, we conclude that the interface roughness becomes more important in deciding the $\left|\eta^{*}\right|$ of the reactive bilayer because the interface roughness of two reactive bilayers was almost the same, although the entanglement effect is not completely excluded.

Figure 3 gives plots of $\left|\eta^{*}\right|$ versus rms roughness for a reactive bilayer $\left(\mathrm{H}_{P S-m C O O H}=\mathrm{H}_{P M M A-G M A}=0.3\right.$ $\mathrm{mm})$. Here, the rms roughness at a given reaction time was measured by AFM. Interestingly, it seems that $\left|\eta^{*}\right|$ is proportional to the rms roughness for larger rms roughness $(>40 \mathrm{~nm})$, whereas a linear relationship is not valid for the smaller rms roughness.

Since the frictional force is related to the interfacial area instead of the interfacial roughness, the relationship between $\mathrm{rms}$ roughness and interfacial area should be known. In this study, we assume that the corrugation of the interface formed by the reaction is qualitatively given as a parallel sinusoidal wave, as shown in Figure 4. Here, the height of the interface (z) is expressed:

$$
z=a \sin \left(\frac{\pi}{b} x\right)
$$

where $a$ is the amplitude of the roughness which is given by $\sqrt{2}$ times rms of the roughness, $b$ is the half of the wavelength of the roughness that is $\sim 100 \mathrm{~nm}$ obtained from AFM image, as shown in Figure $2 b$.
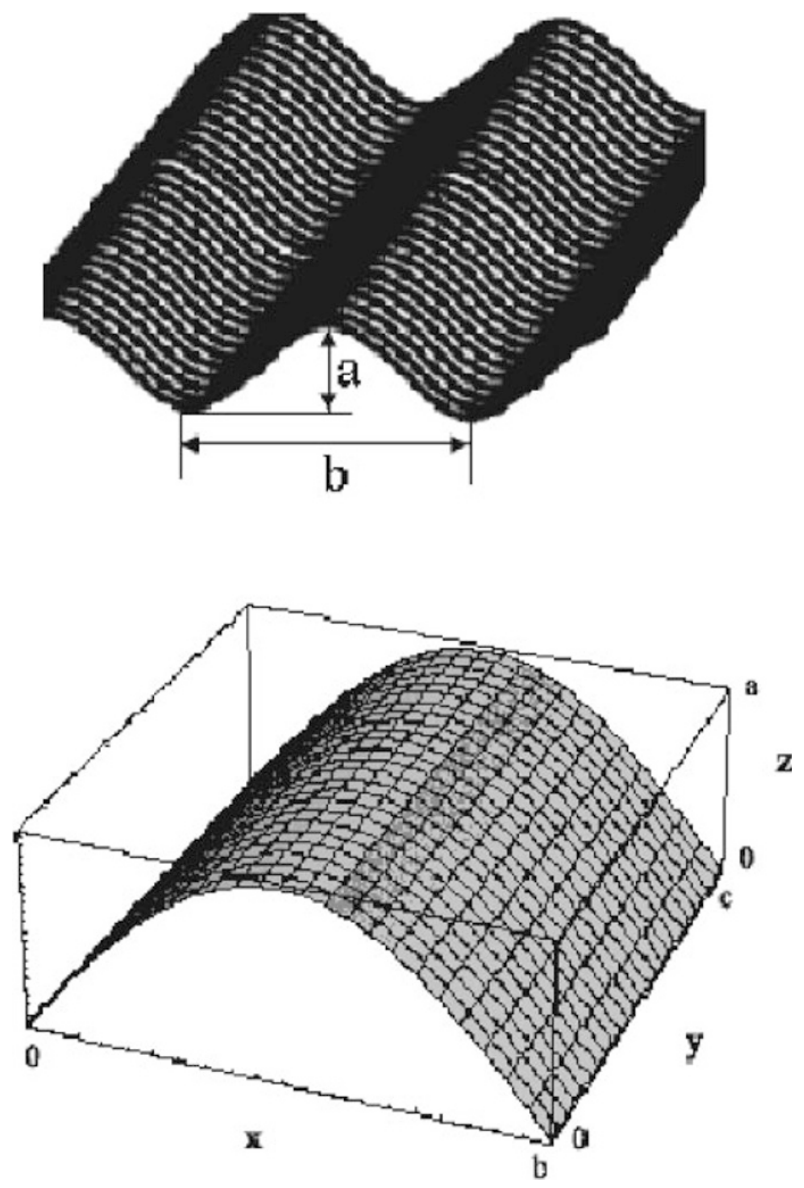

Figure 4. Schematic of roughened interface to calculate the interfacial area.

The curvilinear length $(\mathrm{d} l)$ in $x-z$ plane is given by $\left(d x^{2}+d z^{2}\right)^{1 / 2}$. Therefore, the total interfacial area in unit length $(c)$ is given by

$$
A_{t}=\int_{0}^{c}\left[\int_{0}^{l_{0}} \sqrt{(d x)^{2}+(d z)^{2}}\right] d y
$$

where $l_{0}$ is the curvilinear length at $x=b$. Substitution of eq 6 into eq 7 becomes:

$$
A_{t}=c \int_{0}^{b} \sqrt{1+\left[\frac{a \pi}{b} \cos \left(\frac{\pi}{b} x\right)\right]^{2}} d x
$$

The interfacial area of roughened interface normalized by that $\left(A_{o}=c b\right)$ without roughness as a function of rms roughness is given in Figure 5a. As expected, the interfacial area increased with increasing rms roughness. From the results of Figures 3 and 5a, the plot of $\left|\eta^{*}\right|$ versus $A_{t} / A_{o}$ is given in Figure $5 \mathrm{~b}$. Figure $5 \mathrm{~b}$ shows that the slope of this plot changed dramatically at an $\mathrm{rms}$ roughness of $\sim 40 \mathrm{~nm}$. Notice that the rms roughness of $\sim 40 \mathrm{~nm}$ corresponds to stage II. Thus, we conclude that at stage III where the rms roughness is larger than $40 \mathrm{~nm}$, the increased interfacial area results in additional friction against the flow. Because the interfacial area generated by 
(a)
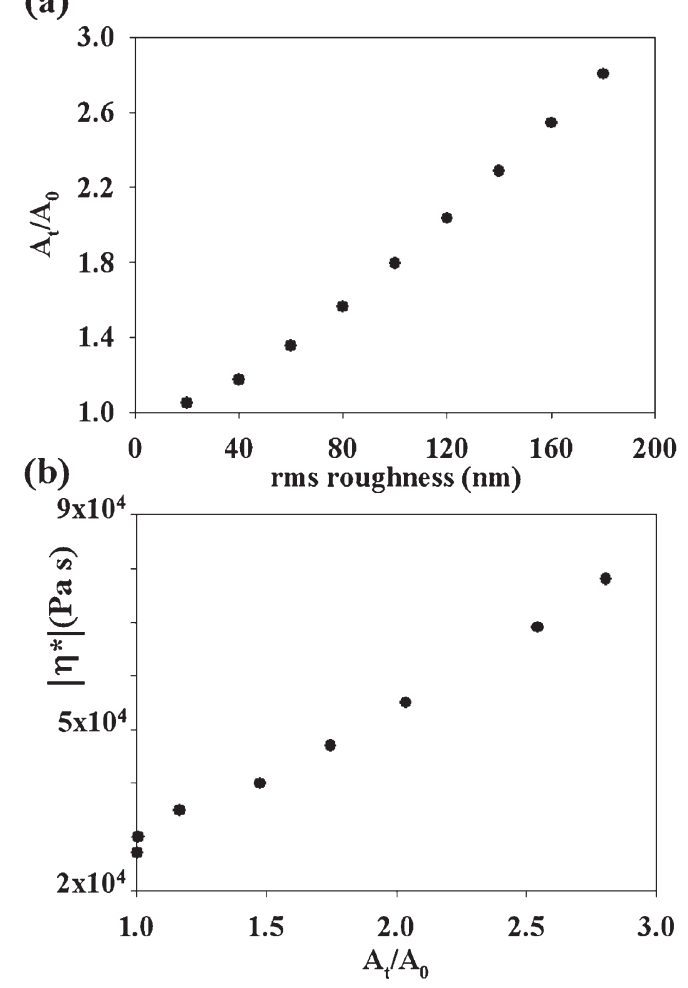

Figure 5. (a) Plot of the normalized interfacial area versus rms roughness estimated by eq 8 . (b) Plot of the $\left|\eta^{*}\right|$ versus the normalized interfacial area.

the interface roughening becomes normal to the flow direction, it might be reasonable to consider that $\left|\eta^{*}\right|$ could increase $\sim 5$ times when the interfacial area was increased to 2.8 times. However, at stage I where the rms roughness is below $40 \mathrm{~nm}$, the increased $\left|\eta^{*}\right|$ could not be explained by the additional friction effect alone.

From the above analysis, we conclude that the additional friction arising from the interfacial roughness is the dominating parameter to increase $\left|\eta^{*}\right|$ of a reactive bilayer at stage III where the rms roughness is larger than $\sim 40 \mathrm{~nm}$. To quantitatively assess the contribution of additional friction effect to the $\left|\eta^{*}\right|$, one needs a pre-made roughened interface. For this purpose, the PS-mCOOH/PMMA-GMA bilayer reacted for $16 \mathrm{~h}$ was pulled out of the rheometer, and selectively rinsed by cyclohexane to remove completely the unreacted PS plate, which produced the PMMA-GMA plate covered with only in situ formed PMMA-graft-PS copolymers. The rms roughness at the top of this PMMA-GMA plate was $\sim 180 \mathrm{~nm}$. Then, we placed a non-reactive PS homopolymer plate with a thickness of $0.3 \mathrm{~mm}$ onto this PMMA-GMA plate. It is noted that $\left|\eta^{*}\right|$ of PS homopolymer was $8 \times 10^{3} \mathrm{Pas}$ at $180^{\circ} \mathrm{C}$, which is the same as that at $180^{\circ} \mathrm{C}$ for PS$\mathrm{mCOOH}$, as seen in Table I. We found that the rms roughness of the interface of this bilayer after the welding two plates for $30 \mathrm{~min}$ at $180^{\circ} \mathrm{C}$ followed by removing the PS plate by rinsing with acetic acid was $\sim 180 \mathrm{~nm}$, indicating that the placement of homo PS plate onto PMMA-GMA plate covered with PMMA-graft-PS copolymers does not change the roughness of the interface. The $\left|\eta^{*}\right|$ of this geometry of the bilayer was $7.8 \times 10^{4} \mathrm{Pas}$, which is almost the same as the $\left|\eta^{*}\right|$ of the reactive bilayer at a reaction time of $16 \mathrm{~h}$, as shown in Figure 1 . However, even in this situation, the entanglement (or stitching) effect between PS chains in PMMA-graft-PS copolymer and PS homopolymer chains should be considered.

Therefore, to exclude completely the entanglement effect, we carried out another experiment with a nonreactive bilayer consisting of PMMA-GMA and polyethylene (PE) with $\left|\eta^{*}\right|$ at $180^{\circ} \mathrm{C}=5 \times 10^{3} \mathrm{~Pa}$ s. It is noted that PMMA and PE as well as PS and PE are highly immiscible; thus it is almost impossible to expect the entanglement between PMMA (or PS) and PE chains near the interface. We prepared two PMMAGMA plates with different roughness: one with a flat surface whose rms roughness is less than $2 \mathrm{~nm}$ prepared by compression molding with Si-wafer, and the other with roughened surface having an rms roughness of $\sim 180 \mathrm{~nm}$ prepared by the same method described above. Subsequently, we placed the PE plate with the flat surface whose rms roughness is less than $2 \mathrm{~nm}$ onto the two different PMMA-GMA plates. For a bilayer with PMMA-GMA with a flat surface, $\left|\eta^{*}\right|$ was $c a$. $10^{4} \mathrm{Pas}$, which is reasonably predicted by eq $2 \mathrm{a}$. However, for another bilayer with PMMA-GMA with roughened surface, $\left|\eta^{*}\right|$ was $c a .3 .4 \times 10^{4} \mathrm{Pas}$, three times larger than that with a flat interface. Therefore, we conclude that the existence of friction effect due to the interface roughness increased significantly the viscosity of a polymer bilayer.

However, to distinguish the effect of the frictional force from the entanglement of polymer chains on the viscosity, we should exclude the latter effect for the PS/PMMA (not PE/PMMA) bilayer system. For this purpose, the PMMA-GMA plate covered with PS-graft-PMMA copolymers and the rms roughness of $\sim 160 \mathrm{~nm}$ was coated by $\mathrm{OsO}_{4}$. Then, we again placed the neat PS homopolymer plate and measured the $\left|\eta^{*}\right|$ after welding two plates for $30 \mathrm{~min}$ at $180^{\circ} \mathrm{C}$. Since the thickness of $\mathrm{OsO}_{4}$ was $c a$. $5 \mathrm{~nm}$, much smaller than rms roughness of PMMA-GMA plate, the effect of this $\mathrm{OsO}_{4}$ layer on $\left|\eta^{*}\right|$ of a bilayer could be negligible. But, this thickness is assumed to be sufficiently large to completely prevent PS chains from crossing over the $\mathrm{OsO}_{4}$ layer to encounter PMMA chains. The $\left|\eta^{*}\right|$ of this bilayer is $6.5 \times 10^{4} \mathrm{Pas}$, smaller than that $\left(7.8 \times 10^{4} \mathrm{Pas}\right)$ without $\mathrm{OsO}_{4}$ layer. We consider that this difference in $\left|\eta^{*}\right|$ between two cases $\left(c a .1 .3 \times 10^{4} \mathrm{Pas}\right)$ results from the entangle- 
ment effect. Therefore, we conclude that the existence of friction effect arising from the interface roughness is much important in determining the viscosity of a reactive bilayer compared with the entanglement of polymer chains neat the interface.

To investigate independently the entanglement effect on $\left|\eta^{*}\right|$ of a bilayer, thin films of PMMA-graftPS copolymer with thicknesses of 50,100, and $200 \mathrm{~nm}$ were spin-coated onto a silicon wafer, and then floated onto PMMA-GMA plate $(0.3 \mathrm{~mm}$ thickness $)$ with a flat surface followed by placing homopolymer PS plate with $0.3 \mathrm{~mm}$ thickness. After the floating of the graft copolymers, we found that the surface of the copolymer film on PMMA-GMA plate was very smooth and the rms of the roughness was less than $2 \mathrm{~nm}$. In this situation, the entanglement effect should be considered since the PS (or PMMA) in the graft copolymer chains can entangle with the homopolymer PS (or homopolymer PMMA) chains. But the roughness effect could be negligible. We found that the $\left|\eta^{*}\right|$ of this bilayer where a thin film of the graft copolymer was sandwiched between two plates was $2.6 \times 10^{4}$ $\mathrm{Pas}$, which is larger than that $\left(1.5 \times 10^{4} \mathrm{Pas}\right)$ of the bilayer consisting of PMMA-GMA and homo PS plates without the graft copolymers. The difference (ca. $1.1 \times 10^{4} \mathrm{Pas}$ ) in $\left|\eta^{*}\right|$ between the two cases is again attributed to the entanglement effect. Interestingly, it was found that the $\left|\eta^{*}\right|$ of the bilayer with thin film of graft copolymer did not change with thickness of the graft copolymer as long as the thickness of graft copolymer ranged from 50 to $200 \mathrm{~nm}$. Furthermore, the rms of the graft copolymers after removing the PS plate was less than $\sim 2 \mathrm{~nm}$. This indicates that the thickness (or the amount) of the graft copolymer themselves does not contribute significantly to a large increase in $\left|\eta^{*}\right|$ of a bilayer as long as the copolymer layer thickness is very small compared with PS plate. Instead, the roughness of the interface becomes more important in determining the $\left|\eta^{*}\right|$ of a bilayer. This further suggests that even for a reactive bilayer with larger amount of in situ formed graft (or block) copolymers, $\left|\eta^{*}\right|$ would not increase much when the rms roughness of the interface is smaller (say, less than $c a .40 \mathrm{~nm}$ ). This is consistent with our previous result $^{33}$ that $\left|\eta^{*}\right|$ of the PS-mCOOH/PMMA-GMA bilayer after applying higher oscillatory frequency of $100 \mathrm{rad} / \mathrm{s}$ is smaller than that of the same bilayer without oscillatory shearing. It is noted that when a higher frequency was applied to this bilayer, the rms roughness of the interface decreased from $c a .180 \mathrm{~nm}$ to ca. $20 \mathrm{~nm}$, although the amount of in situ formed graft copolymers under the oscillatory shearing was almost the same as that without shearing.

To summarize all results, the $\left|\eta^{*}\right|$ of a reactive bilayer can be expressed as follows:

$$
\begin{aligned}
\eta^{*}{ }_{\text {total }}= & \left(\frac{\varphi_{P S-m \mathrm{COOH}}}{\eta^{*} P S-m \mathrm{COOH}}+\frac{\varphi_{P M M A-G M A}}{\eta^{*} P S-m \mathrm{COOH}}\right)^{-1} \\
& +F_{\text {entangle }}(\delta, \cdots)+F_{\text {friction }}(\delta, \cdots)
\end{aligned}
$$

where $F_{\text {entangle }}$ and $F_{\text {friction }}$ represent the increase of $\left|\eta^{*}\right|$ due to the entanglement (or stitching) effect and the additional friction, respectively. And $\delta$ is the rms roughness of the interface. We found that $F_{\text {friction }}$ becomes the dominant factor in deciding $\left|\eta^{*}\right|$ for $\delta>40 \mathrm{~nm}$, whereas $F_{\text {entangle }}$ becomes important at lower rms roughness.

Finally, we investigated the change of $\left|\eta^{*}\right|$ with increasing number of reactive layer, and the result is given by Figure 6. For this purpose, we maintained the total thickness of plates to be $1.0 \mathrm{~mm}$ with the same thickness of each plate. Namely, when the number of the interface was three, the film geometry was PMMA-GMA/PS/PMMA-GMA/PS with each plate thickness of $0.25 \mathrm{~mm}$. It is seen in Figure 6 that $\left|\eta^{*}\right|$ before the reaction are essentially the same for three cases, which can be predicted by eq 2a. Also, the trend of the increase of $\left|\eta^{*}\right|$ seemed to be similar, but the $\left|\eta^{*}\right|$ became larger, as the number of the interface increases. This result can be explained by increased total interfacial area. Interestingly, $\left|\eta^{*}\right|$ seems to approach to a limiting value with increasing number of the interface. We speculated that the asymptotic value would be related to the viscosity of the same polymer blend prepared by mechanical mixer, which generates enormous amount of the interface. We prepared reactive blending of 50/50 (wt/wt) PS-

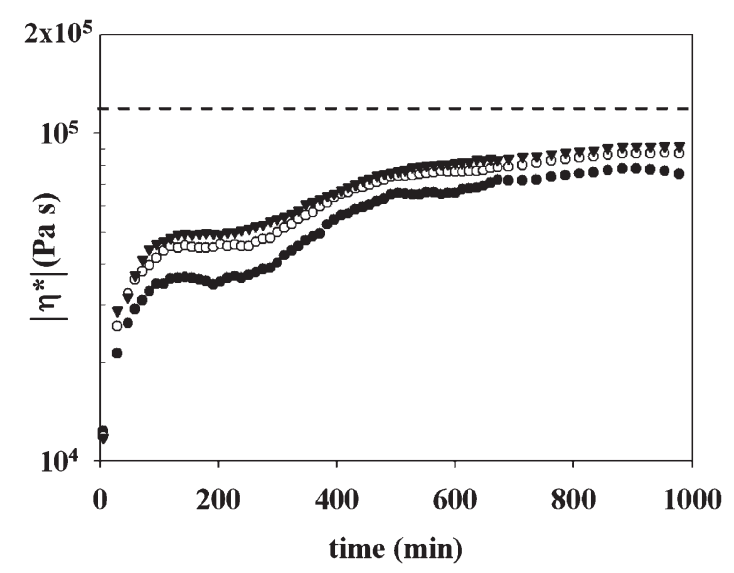

Figure 6. Plot of $\left|\eta^{*}\right|$ at $\omega=0.1 \mathrm{rad} / \mathrm{s}$ and $180^{\circ} \mathrm{C}$ versus reaction time for symmetric PS-mCOOH $(0.5 \mathrm{~mm}) / \mathrm{PMMA}-\mathrm{GMA}$ $(0.5 \mathrm{~mm})$ bilayer $(\bullet)$, PMMA-GMA $(0.25 \mathrm{~mm}) / \mathrm{PS}-\mathrm{mCOOH}$ $(0.5 \mathrm{~mm})$ /PMMA-GMA $(0.25 \mathrm{~mm})$ three layers $(\bigcirc)$, and PMMAGMA $(0.25 \mathrm{~mm}) / \mathrm{PS}-\mathrm{mCOOH} \quad(0.25 \mathrm{~mm}) / \mathrm{PMMA}-\mathrm{GMA} \quad(0.25$ $\mathrm{mm}) / \mathrm{PS}-\mathrm{mCOOH}(0.25 \mathrm{~mm})$ four layers $(\boldsymbol{\nabla})$. The dashed line represents $\left|\eta^{*}\right|$ for 50/50 (w/w) PS-mCOOH/PMMA-GMA blend prepared by a Mini-Max molder with shear rate of $20 \mathrm{~s}^{-1}$ at $220^{\circ} \mathrm{C}$ for $20 \mathrm{~min}$. 
mCOOH/PMMA-GMA by using a MiniMax molder with a shear rate of $20 \mathrm{~s}^{-1}$ at $220^{\circ} \mathrm{C}$ and for $20 \mathrm{~min}$. The $\left|\eta^{*}\right|$ of this reactive blend was measured as $1.1 \times$ $10^{5} \mathrm{Pas}$, twice larger than that for a four-layered sample. We consider that when the number of layer is large enough, the $\left|\eta^{*}\right|$ of multilayered sample would approach the $\left|\eta^{*}\right|$ of a blend prepared by a mixer.

\section{CONCLUSION}

In this study, we have shown that the rheological properties of a reactive bilayer consisting of PS$\mathrm{mCOOH}$ and PMMA-GMA depended on the rms roughness of the interface in addition to the existence of in situ formed graft copolymers at the interface. We found that the $\left|\eta^{*}\right|$ of the reactive bilayer was unusually higher than the predicted value from the inverse relationship which holds for a non-reactive polymer bilayer, even though the interfacial roughness generated from graft copolymers formed in situ from the reaction near the interface was much smaller than the thicknesses of two plates of PS-mCOOH and PMMA-GMA. However, when the rms roughness of the interface is very small (less than $40 \mathrm{~nm}$ ), the entanglement effect becomes an important parameter in determining rheological properties.

Acknowledgment. This work was supported by the National Creative Research Initiative Program supported by Korea Organization of Science and Engineering Foundation.

\section{REFERENCES}

1. D. R. Paul and S. Newman, "Polymer Blends," Academic Press, New York, 1978, vol. 1.

2. L. A. Utracki, "Polymer Alloys and Blends," Hanser, New York, 1989.

3. C. D. Han and T. C. Yu, J. Appl. Polym. Sci., 15, 1163 (1971).

4. L. A. Utracki and M. R. Kamal, Polym. Eng. Sci., 22, 96 (1982).

5. N. Alle and J. Lyngaae-Jorgensen, Rheol. Acta, 19, 94 (1980).

6. Y. C. Kim, S. J. Lee, J. C. Kim, and H. Cho, Polym. J., 37, 206 (2005).

7. C. D. Han and T. C. Yu, Polym. Eng. Sci., 12, 81 (1972).

8. C. K. Shih, Polym. Eng. Sci., 16, 742 (1976).

9. S. Danesi and S. R. Porter, Polymer, 19, 448 (1978).

10. Y. S. Lipatov, V. F. Shumsky, A. N. Gorbatenko, Y. N. Panov, and L. S. Bolotnikova, J. Appl. Polym. Sci., 26,
499 (1981).

11. a) C. D. Han and H. H. Yang, J. Appl. Polym. Sci., 33, 1199 (1987).

b) C. D. Han and H. H. Yang, J. Appl. Polym. Sci., 33, 1221 (1987).

12. a) C. D. Han and J. K. Kim, Macromolecules, 22, 1914 (1989).

b) C. D. Han and J. K. Kim, Macromolecules, 22, 4292 (1989).

13. C. D. Han, H. S. Chung, and J. K. Kim, Polymer, 33, 546 (1992)

14. J. K. Kim, C. D. Han, and Y. J. Lee, Polym. J., 24, 205 (1992).

15. C. D. Han, "Multiphase Flow in Polymer Processing," Academic Press, New York, 1981.

16. N. Grizzuti, G. Buonocore, and G. Iorioc, J. Rheol., 44, 149 (2000).

17. L. A. Utracki, "Commercial Polymer Blends," Chapman\& Hall, New York, 1998.

18. I. K. Andrea, A. P. Anastasia, TH. G. Stella, M. MarieClaude, V.-M. Claire, and V. N. Anastasios, Electroanalysis, 18, 456 (2006).

19. W. M. Diffey, R. H. Trimm, M. G. Temmen, and P. R. Ashley, J. Lightwave Technol., 23, 1787 (2005).

20. K. Lamnawar and A. Maazouz, Rheol. Acta, 45, 411 (2006).

21. R. F. Heitmiller, R. Z. Naar, and H. H. Zabusky, J. Appl. Polym. Sci., 8, 873 (1964).

22. J. Zhao, L. Mascia, and V. Nassehi, Adv. Polym. Technol., 16, 209 (1997).

23. R. Zhao and C. W. Macosko, J. Rheol., 46, 145 (2002).

24. H. Y. Kim, U. Jeong, and J. K. Kim, Macromolecules, 36, 1594 (2003).

25. X. Yu, Y. Wu, B. Li, and Y. Han, Polymer, 46, 3337 (2005).

26. J. K. Kim and H. Lee, Polymer, 37, 305 (1996).

27. H. K. Jeon and J. K. Kim, Macromolecules, 31, 9273 (1998).

28. a) H. K. Jeon and J. K. Kim, Polymer, 39, 6227 (1998).

b) H. K. Jeon and J. K. Kim, Korea. Polym. J., 7, 124 (1999).

29. K. K. Jeon, H. T. O, and J. K. Kim, Polymer, 42, 3259 (2001).

30. H. K. Jeon and J. K. Kim, Macromolecules, 33, 8200 (2000).

31. H. Y. Kim, D. Y. Ryu, U. Jeong, D. H. Kho, and J. K. Kim, Macromol. Rapid Commun., 26, 1428 (2005).

32. D. H. Kho, S. H. Chae, U. Jeong, H. Y. Kim, and J. K. Kim, Macromolecules, 38, 3820 (2005).

33. H. Y. Kim, D. H. Lee, and J. K. Kim, Polymer, 47, 5108 (2006).

34. S. Y. Yang, I. Ryu, H. Y. Kim, J. K. Kim, S. K. Jang, and T. P. Russell, Adv. Mater., 18, 709 (2006).

35. U. Jeong, D. Y. Ryu, J. K. Kim, D. H. Kim, T. P. Russell, and C. J. Hawker, Adv. Mater., 15, 1247 (2003).

36. D. Graebling, R. Muller, and J. F. Palierne, Macromolecules, 26, 320 (1993).

37. J. F. Palierne, Rheol. Acta, 29, 204 (1990). 